\title{
A Novel Case-Finding Instrument for Chronic Obstructive Pulmonary Disease in Low- and Middle-Income Country Settings
}

This article was published in the following Dove Press journal: International Journal of Chronic Obstructive Pulmonary Disease

\author{
Trishul Siddharthan $\mathbb{D}^{1,2}$ \\ Adaeze C Wosu ${ }^{2,3}$ \\ Suzanne L Pollard ${ }^{1,2}$ \\ Shakir Hossen ${ }^{1,2}$ \\ Patricia Alupo (1D) \\ Timothy Shade ${ }^{1,2}$ \\ Robert Kalyesubula (iD ${ }^{4}$ \\ Shumonta Quaderi ${ }^{6}$ \\ Robert A Wise (D) \\ John R Hurst ${ }^{6}$ \\ Bruce Kirenga ${ }^{4,5}$ \\ William Checkley ${ }^{1,2}$ \\ On behalf of LiNK Cohort \\ Study Investigators \\ 'Division of Pulmonary and Critical Care, \\ School of Medicine, Johns Hopkins \\ University, Baltimore, MD, USA; ${ }^{2}$ Center \\ for Global Non-Communicable Disease \\ Research and Training, Johns Hopkins \\ University, Baltimore, MD, USA; \\ ${ }^{3}$ Department of Epidemiology, \\ Bloomberg School of Public Health, Johns \\ Hopkins University, Baltimore, MD, USA; \\ ${ }^{4}$ School of Medicine, College of Health \\ Sciences, Makerere University, Kampala, \\ Uganda; ${ }^{5}$ Makerere Lung Institute, \\ Makerere University, Kampala, Uganda; \\ ${ }^{6} \mathrm{UCL}$ Respiratory, University College \\ London, London, UK
}

Correspondence: William Checkley Division of Pulmonary and Critical Care, School of Medicine, Johns Hopkins University, 1800 Orleans Ave Suite 9121, Baltimore, MD 21287, USA

Tel + I 443-287-4587

Email wchecklI@jhmi.edu
Background: Low- and middle-income countries (LMICs) account for $>90 \%$ of deaths and illness episodes related to COPD; however, this condition is commonly underdiagnosed in these settings. Case-finding instruments for COPD may improve diagnosis and identify individuals that need treatment, but few have been validated in resource-limited settings.

Methods: We conducted a population-based cross-sectional study in Uganda to assess the diagnostic accuracy of a respiratory symptom, exposure and functional questionnaire in combination with peak expiratory flow for COPD diagnosis using post-bronchodilator $\mathrm{FEV}_{1} / \mathrm{FVC}$ z-score below the 5 th percentile as the gold standard. We included locally relevant exposure questions and statistical learning techniques to identify the most important risk factors for COPD. We used $80 \%$ of the data to develop the case-finding instrument and validated it in the remaining $20 \%$. We evaluated for calibration and discrimination using standard approaches. The final score, COLA (COPD in LMICs Assessment), included seven questions, age and pre-bronchodilator peak expiratory flow.

Results: We analyzed data from 1,173 participants (average age 47 years, $46.9 \%$ male, $4.5 \%$ with COPD) with acceptable and reproducible spirometry. The seven questions yielded a cross-validated area-under-the-curve [AUC] of 0.68 (95\% CI 0.61-0.75) with higher scores conferring greater odds of COPD. The inclusion of peak expiratory flow and age improved prediction in a validation sample (AUC $=0.83,95 \%$ CI $0.78-0.88$ ) with a positive predictive value of $50 \%$ and a negative predictive value of $96 \%$. The final instrument (COLA) included seven questions, age and pre-bronchodilator peak expiratory flow.

Conclusion: COLA predicted COPD in urban and rural settings in Uganda has high calibration and discrimination, and could serve as a simple, low-cost screening tool in resource-limited settings.

Keywords: low- and middle-income countries, COPD, COLA, respiratory symptom

\section{Introduction}

Chronic obstructive pulmonary disease (COPD) is a common and preventable disease characterized by persistent respiratory symptoms and airflow limitation. In 2015, it was estimated that 174 million people worldwide had clinically significant COPD, and an estimated 3.2 million individuals died from the disease. ${ }^{1}$ Over $90 \%$ of morbidity and mortality related to COPD occurs in low- and middleincome countries (LMICs), where the primary risk factor for COPD is household air pollution secondary to biomass burning for cooking and heating, although the prevalence of tobacco smoking is increasing rapidly in these regions. ${ }^{2}$ COPD is 
expected to become the third leading cause of death by 2030 and COPD under diagnosis has been documented to be universally high. ${ }^{3}$

Diagnosis of COPD by post-bronchodilator spirometry requires trained technicians to perform the test, and pulmonary clinicians to interpret the tests who, if available, are often localized to a few urban cities in LMIC settings. ${ }^{4}$ Additionally, spirometry is resource-intensive to implement across most existing LMIC health systems. COPD case-finding questionnaires have been used in high-income settings to identify individuals at risk of COPD in primary care clinics for further testing with diagnostic spirometry. ${ }^{5-7}$ These self-reported instruments rely on symptoms and risk factors related to COPD and have been validated among clinic-based populations with moderately high predictive value. In 2010, Yawn et al developed and validated a simple five-item Lung Function Questionnaire (LFQ) and compared this to COPD diagnosis by spirometry (area under the curve $[\mathrm{AUC}]=0.720$ with sensitivity (SN) and specificity (SP) of $73.2 \%$ and $58.2 \%$, respectively). ${ }^{7}$ Martinez et al combined peak expiratory flow (PEF) measurements with a case-finding instrument and documented improved SN (89.7\%), SP (93.1\%), and AUC for detecting COPD (0.91). ${ }^{5}$ No instruments to date have been validated in LMIC settings, which have a different profile of risk factors and presentation of chronic airflow obstruction. Additionally, these questionnaires offer a simple and potentially cost-effective method of identifying individuals for definitive testing and/or treatment in these settings.

We sought to develop a case-finding questionnaire in an LMIC setting, including an assessment of PEF based on previously validated questions used in other case-finding surveys, and validate its use to identify individuals at risk for COPD. ${ }^{5,7}$

\section{Methods}

\section{Study Setting}

We conducted a cross-sectional analysis of data collected at baseline for the Lung Function in Nakaseke and Kampala Study (LiNK) cohort in a rural and an urban sample in Uganda between November 2015 and June 2016. ${ }^{8}$ The urban sample was drawn from Kampala, the capital of Uganda, with a population of 1.5 million in an estimated 416,070 households. ${ }^{9}$ The rural sample was drawn from Nakaseke, a health district with 43,167 households and an estimated population of $208,500 .{ }^{9,10}$ A total of 1,502 participants had acceptable and reproducible spirometry in the LiNK cohort. We included respiratory symptoms and functional questionnaires among a subset of participants, administered at time of spirometry, which are included in this analysis (Supplement Data Table 1). As part of this ancillary study, we aimed to assess the diagnostic value of a questionnaire with multiple domains including respiratory symptoms, functional status, and relevant exposures for identification of obstruction on spirometry.

\section{Study Design}

Twenty-five enumerated areas each were selected from Nakaseke and Kampala using probability proportional to population size. Inclusion criteria included age $\geq 35$ years and less than 95 years, full-time residency in either Kampala or Nakaseke, and capacity to consent to the study. Exclusion criteria included having active pulmonary tuberculosis, a current respiratory infection, or being pregnant. The study was approved by the Institutional Review Boards of Mulago Hospital and the Uganda National Council for Science and Technology in Kampala, Uganda and the Johns Hopkins School of Medicine in Baltimore, USA. ${ }^{8}$ All participants provided informed consent, and this study was conducted in accordance with the Declaration of Helsinki.

\section{Procedures}

Anthropometric measurements were taken in triplicate and demographic questionnaires including biomass fuel smoke exposure, cigarette smoking, HIV status, and history of pulmonary tuberculosis were administered. Standardized respiratory symptom questionnaires modified from the BOLD questionnaire were applied in Luganda, the local language in the study areas. ${ }^{2}$ Spirometry was conducted using the Easy-On-PC spirometer (ndd, Zurich, Switzerland) according to the ATS/ERS guidelines. ${ }^{11}$ Post-bronchodilator testing (400 mcg of inhaled salbutamol via a spacer) was performed on all participants with evidence of obstruction defined by the NHANES African American reference. ${ }^{12}$ Participants with low-quality spirometry were asked to repeat the test on another day for up to three attempts.

\section{Definitions}

Household size and secondary education were used as proxies for socioeconomic status. We defined exposure to biomass fuel smoke as household use of wood or charcoal as primary fuel for cooking or heating; daily smoking as selfreport of smoking one or more cigarettes per day; 
urbanization as residing in Kampala; underweight as a body mass index $(\mathrm{BMI})<18.5 \mathrm{~kg} / \mathrm{m}^{2}$ and obesity as a $\mathrm{BMI} \geq$ $30 \mathrm{~kg} / \mathrm{m}^{2}$. We defined COPD as a post-bronchodilator $\mathrm{FEV}_{1}$ /FVC ratio Z-score $\leq-1.64$ standard deviations using the NHANES African American reference population and bronchodilator reversibility as a post-bronchodilator increase in FEV1 of $12 \%$ or more from baseline or an increase in FVC of $200 \mathrm{~mL}$ or more. ${ }^{13}$ Peak expiratory flow measurements were obtained from pre-bronchodilator spirometry.

\section{Risk Factors}

We previously conducted a comprehensive literature search and interviews with patients and providers in Uganda to identify 21 risk factors for COPD (Figure 1). ${ }^{3,5-7}$ Questions included demographic background (3 questions), exposure history (3 questions), respiratory symptoms (11 questions), medication use (1 question), family history (1 question) and functional status (2 questions).

\section{Biostatistical Methods}

The primary objective was to test the diagnostic accuracy of a multi-domain questionnaire that included questions about respiratory symptoms, functional status, personal exposures, and age in combination with peak expiratory flow to identify participants with COPD in a Ugandan community setting. We first divided the dataset into a test set consisting of $80 \%$ of participants selected using a simple random sample, and a validation set consisting of the remaining $20 \%$. We then conducted a random forest analysis using 1000 bootstrap samples and five variables randomly sampled as candidates for each tree to identify the five most important respiratory

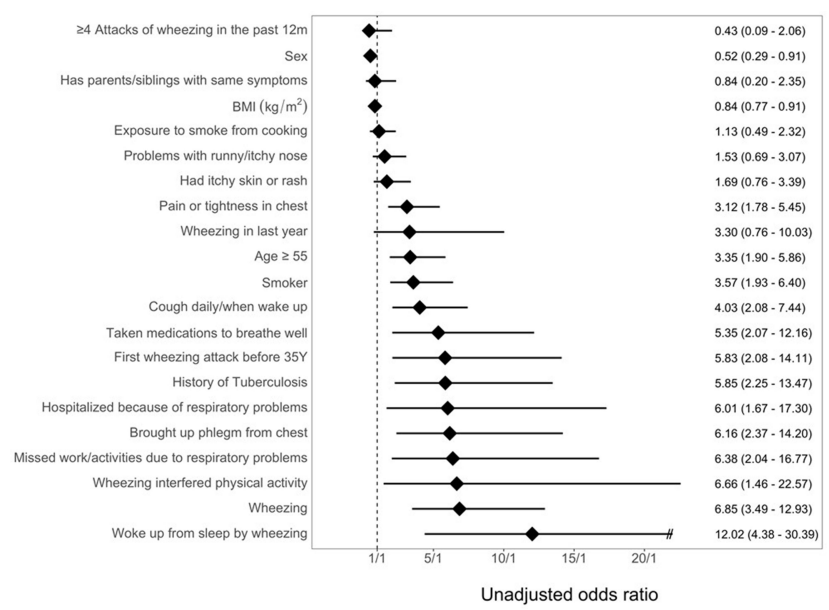

Figure I Odds of COPD by risk factor. Unadjusted odds of COPD by risk factor with $95 \% \mathrm{Cl}$. symptoms that identify COPD in the training set. ${ }^{14} \mathrm{We}$ added smoking and biomass exposure variables a priori to ensure generalizability with other LMIC settings. We utilized conditional inference trees and graphically determined cutoff values for age and peak expiratory flow most predictive of airflow obstruction on spirometry. The final casefinding instrument, COLA (COPD in LMICs Assessment), consisted of seven questions, age and peak expiratory flow values. We then developed a scoring system whereby participants received 1-point for each of the seven respiratory symptoms, exposure and functional questions (if present), 1-point if the participant was $\geq 55$ years of age, 1-point if peak expiratory flow was between 250 and $399 \mathrm{~L} / \mathrm{min}$, and 2-points if peak expiratory flow was less than $250 \mathrm{~L} / \mathrm{min}$ (Table 1).

Using the test set, we used bias reduced logistic regression to determine the odds of having COPD as a function of the COLA score. ${ }^{15}$ Bias reduction was used given the low number of individuals with COPD in each score bin. To assess calibration of our prediction model, we plotted the observed against predicted prevalence of COPD across the range of values of the COLA scores. ${ }^{16}$ We then tested our prediction model using k-fold cross-validation on the validation set. To assess discrimination, we estimated the AUC of the model in the validation set. We used standard measures to measure the diagnostic accuracy of our risk

Table I Derived COLA Score

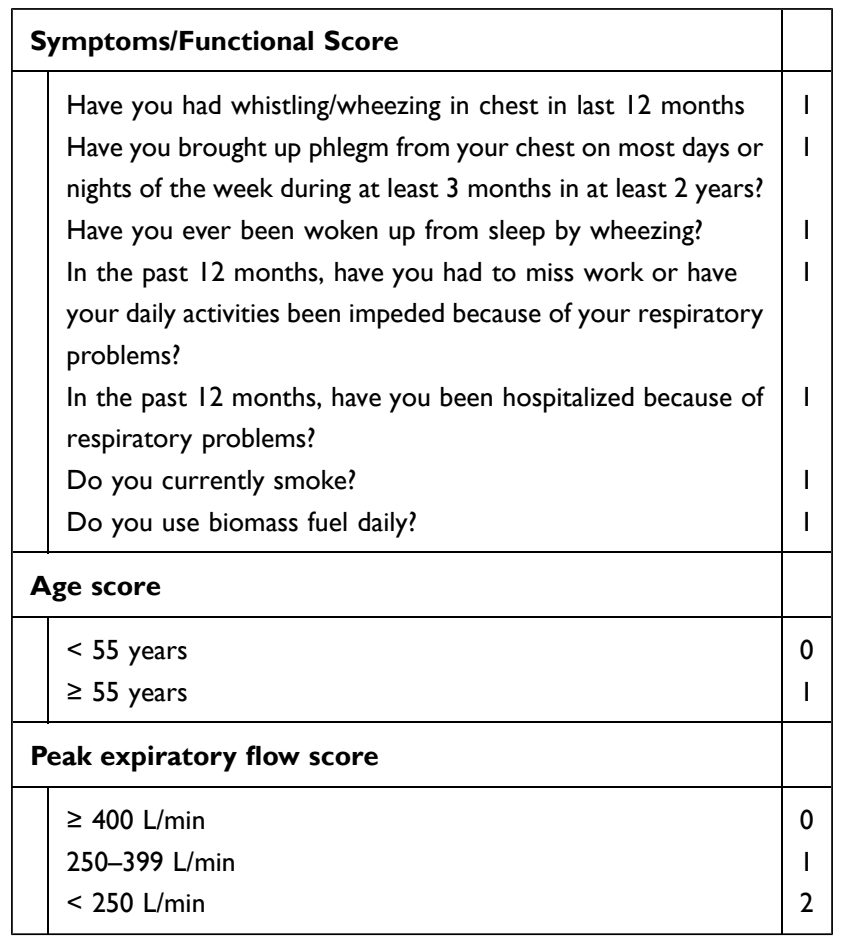


score and peak expiratory flow including sensitivity, specificity, and AUC on the test set using post-bronchodilator spirometry values as the gold standard. Analyses were conducted in $\mathrm{R}$ version 3.6.2 (Dark and Stormy Night) using the brglm, party and caret packages (www-r.project.org).

\section{Results}

\section{Demographic Characteristics}

Of 1,173 participants who were enrolled with acceptable and reproducible spirometry and complete respiratory symptom data, the mean $( \pm \mathrm{SD})$ age was $47.01 \pm 10.6$ years and $46.9 \%$ were male $(59.8 \%$ in rural, $40.3 \%$ in urban). 329 participants were excluded due to incomplete data. Self-reported biomass fuel smoke exposure was high in both settings, with the rural setting almost exclusively using biomass fuels for cooking (99.6\%). We summarized demographic characteristics of individuals without COPD $(n=1,120)$ and with COPD $(n=53)$ in Table 2. Current smoking, age $\geq 55$ years, taking medications for shortness of breath, and history of tuberculosis were associated with COPD. A range of respiratory symptoms including cough daily/upon waking; first wheezing attack before age 35 years; hospitalization due to respiratory illness in past year; phlegm on most days over three months in the past year; missed work activities due to respiratory illness in the past year; wheezing in the past year; wheezing interfering with physical activity; and, nocturnal awakening due to wheezing were also associated with COPD (Figure 1).

\section{Building a Risk Score for COPD}

Our random forest analyses revealed that the most important combination of variables for the identification of COPD were: nocturnal awakening with wheezing; phlegm on most days over three months in the past year; wheezing in the past year; hospitalization due to respiratory symptoms in the past year; and, missed work or limitation due to respiratory illness in the past year. We included the additional variables of current biomass exposure and daily cigarette smoking to include relevant exposures across other LMIC settings (Table 1$)^{2}$

Performance of PEF alone was evaluated in relationship to obstruction on spirometry in the training set. We also graphically compared peak expiratory flow using 50$\mathrm{mL}$ increments to determine optimal cutoffs for differentiating cases from controls (Supplement Figure 1). We compared this to estimates generated by conditional inference trees $(266 \mathrm{~L} / \mathrm{min}$ and $330 \mathrm{~L} / \mathrm{min})$ to identify optimum values $(250 \mathrm{~L} / \mathrm{min}$ and $400 \mathrm{~L} / \mathrm{min})$ for inclusion in our final model.

Because there were too few participants at the extremes of the COLA total scores to derive meaningful operating characteristics of the instruments, we grouped COLA total scores into five risk scores as follows: 1 (0-1 points), 2 (2 points), 3 (3 points), 4 (4 points), and 5 (greater than 5 points). Calibration against airflow obstruction on spirometry demonstrated that prevalence of COPD on the training set increased with COLA risk score, and that predicted values were similar to observed values (Figure 2). A risk score of one $(n=465)$ conferred an observed probability of COPD of $0.5 \%$ and an estimated probability of $0.8 \%$. A risk score of five $(\mathrm{n}=20)$ resulted in an observed probability of COPD of $50 \%$ and the highest estimated probability of COPD (52.7\%).

\section{Diagnostic Accuracy}

Age and seven questions yielded a cross-validated AUC of 0.74 (95\% CI 0.67-0.81), whereas PEF and seven questions yielded a cross-validated AUC of 0.83 (95\% CI 0.78-0.89). The COLA score improved overall discrimination $(0.83,95 \%$ CI $0.78-0.88)$ when compared to the latter scores (Figure 3). The cross-validated AUC was similar to the non-cross validated AUC ( 0.83 vs 0.83 ), suggesting that the logistic model was not overfitted. We additionally conducted analysis to assess the validity of a truncated score (six questions, avoiding a duplicate question relating to wheeze), which demonstrated similar operating characteristics (AUC 0.82 ). A COLA score $\geq 5$ yielded the highest combination of sensitivity, specificity and positive predictive value (Table 3).

\section{Discussion}

We found that seven questions related to respiratory symptoms, functional status and exposure history when combined with PEF and age had good discrimination for COPD in a LMIC setting. The most predictive domains for identifying COPD included age, daily cough and production of phlegm. Our data suggest that questionnaires combined with PEF measurements are valid instruments for identifying individuals with COPD and provide a potential simple and low-cost screening tool for case finding in resource-limited settings. 
Table 2 Baseline Characteristics of I,I73 LiNK Study Participants with Complete Respiratory Symptom Data

\begin{tabular}{|c|c|c|c|c|}
\hline Variables & $\mathbf{N}$ & $\begin{array}{l}\text { COPD } \\
(n=53)\end{array}$ & $\begin{array}{l}\text { No COPD } \\
(n=I, I 20)\end{array}$ & P-value \\
\hline Socio-demographics & 1173 & & & \\
\hline Age, in years: Mean \pm SD & 1173 & $54.1(12.2)$ & $46.7(10.3)$ & $<0.01$ \\
\hline Sex: Female & 622 & $20(37.7 \%)$ & $602(53.8 \%)$ & 0.03 \\
\hline Head of house: Yes & 855 & $46(86.8 \%)$ & $809(72.3 \%)$ & 0.03 \\
\hline Working: Yes & 1066 & 47 (88.7\%) & $1019(91.1 \%)$ & 0.72 \\
\hline Marital status, n (\%) & 1173 & & & 0.63 \\
\hline Single & 150 & 7 (I3.2\%) & $14 \mid(12.6 \%)$ & \\
\hline Married & 742 & $35(66 \%)$ & $706(63.1 \%)$ & \\
\hline Cohabiting & 67 & $2(3.8 \%)$ & $64(5.8 \%)$ & \\
\hline Separated/Divorced & 93 & $2(3.8 \%)$ & $90(8.1 \%)$ & \\
\hline Widow/Widower & 108 & 7 (I3.2\%) & 101 (9\%) & \\
\hline \multicolumn{5}{|l|}{ Household characteristics } \\
\hline \multirow{3}{*}{$\begin{array}{l}\text { Household size, median (IQR) } \\
\text { Has chimney, n (\%) } \\
\text { Current Biomass use, n (\%) }\end{array}$} & 1173 & $4(2,6)$ & $5(3,7)$ & 0.32 \\
\hline & 243 & 17 (32.1\%) & $226(20.3 \%)$ & 0.06 \\
\hline & 1140 & $53(100 \%)$ & 1087 (97.1\%) & 0.40 \\
\hline Cooking location, n (\%) & & & & 0.07 \\
\hline Outside the house & 576 & $23(43.4 \%)$ & $553(49.4 \%)$ & \\
\hline Inside the main living area & 102 & I (I.9\%) & 101 (9\%) & \\
\hline In a separate room/hut & 494 & $29(54.7 \%)$ & 465 (4I.6\%) & \\
\hline Fuel source & & & & $<0.01$ \\
\hline Wood & 690 & $44(83 \%)$ & $646(57.7 \%)$ & \\
\hline Coal & 450 & $9(17 \%)$ & $44 I(39.4 \%)$ & \\
\hline Other & 33 & $0(0)$ & $33(2.9 \%)$ & \\
\hline \multicolumn{5}{|l|}{ Smoking status } \\
\hline Daily Smoker & 97 & $10(18.9 \%)$ & 87 (7.8\%) & 0.01 \\
\hline Pack-years: Median (IQR) & 1173 & $0(0,1.5)$ & $0(0,0)$ & $<0.01$ \\
\hline Pack-years: Mean \pm SD & 1173 & $2.1(4.8)$ & $0.8(3.6)$ & 0.01 \\
\hline \multicolumn{5}{|l|}{ Lung function, median z-score (IQR) } \\
\hline Pre-bronchodilator FEV & 1173 & $-1.95(-2.30,-1.60)$, & $-0.30(-0.37,-0.24)$ & $<0.01$ \\
\hline Pre-bronchodilator FVC & 1173 & $-0.57(-1.00,-0.15)$ & $-0.27(-0.33,-0.20)$ & 0.07 \\
\hline Pre-bronchodilator FEV/FVC ratio & 1173 & $-3.43(-3.85,-3.00)$ & $-0.10(-0.15),-0.04$ & $<0.01$ \\
\hline Peak Expiratory Flow & 1173 & $290(258,322)$ & $432(426,439)$ & $<0.01$ \\
\hline Body mass index, mean (SD) & 1173 & $21.8(4.7)$ & $24.9(5)$ & $<0.01$ \\
\hline History of pulmonary tuberculosis, n (\%) & 1173 & 7 (I5.2\%) & $30(3 \%)$ & $<0.01$ \\
\hline
\end{tabular}

A number of case-finding instruments have been validated in high-income settings. ${ }^{5-7}$ Yawn et al utilized data from the third National Health and Nutrition Examination Survey to develop a five-item lung function questionnaire (LFQ) including age, smoking status and respiratory symptoms. ${ }^{7}$ The model with all items had an AUC of 0.72 with SN and SP of $73.2 \%$ and
$58.2 \%$, respectively. The authors found that participants above the age of 50 years had the highest odds for having COPD $(\mathrm{OR}=3.32,95 \% \mathrm{CI}=1.87-5.90)$, followed by dyspnea $(\mathrm{OR}=1.99, \mathrm{CI}=1.22-3.27)$ and smoking history $(\mathrm{OR}=1.81, \mathrm{CI}=1.33-2.88) .^{7}$ Participants among the current cohort who stated respiratory symptoms (ie wheeze, phlegm and cough) 


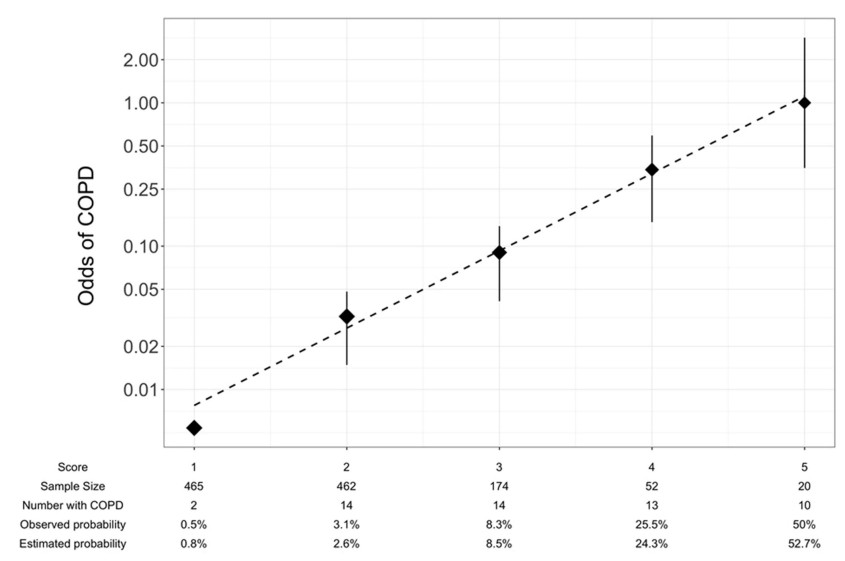

Figure 2 Calibration of generated model on test set. Log odds of COPD compared to 5-point respiratory symptom and exposure selection, age and PEF from random forest analysis with best-fit line.

had higher odds of COPD compared to previous studies suggesting that respiratory symptoms may have higher discriminatory value in low- and middle-income settings. Individuals with COPD in LMICs are largely not on maintenance therapy for respiratory symptoms and respiratory symptoms may be more pronounced between groups. Furthermore some have suggested that COPD found among non-smokers may represent a distinct endotype, with a different presentation compared to COPD from cigarette exposure. ${ }^{17,18}$ The LFQ was subsequently validated in a clinic based sample with a lower AUC (0.65) and specificity (47.8\%), though with a higher sensitivity (82.6\%). ${ }^{19}$ Among a population-based sample of 9,390 participants, the BOLD investigators found among those with moderate/severe COPD, use of questionnaire data alone permitted high sensitivity (97\%) but required confirmatory spirometry in $80 \%$ of participants. ${ }^{20}$ The COLA score demonstrated a higher AUC than studies in high-income settings, which is a result of the higher discriminatory value of respiratory symptoms in differentiating individuals with COPD in the LMIC cohort.

Martinez et al used random forest analysis to identify five items (CAPTURE) with the highest operating characteristics and combined this with PEF. ${ }^{5}$ Notably, the CAPTURE questionnaire did not include age or smoking status in the final candidate selection. While the CAPTURE questionnaire was comparable to the 5-item LFQ in its ability to differentiate people with COPD from those without COPD (AUC 0.71 vs 0.795 ), the addition of PEF to this questionnaire improved discrimination for COPD (SN 89.7\%, SP 78.1\%, AUC 0.906). Both the LFQ and CAPTURE questionnaires were validated against a primary care, clinic-based sample in a high-income country (HIC) setting. COPD surveys administered in HIC primary care settings are neither feasible nor appropriate in many LMIC settings, where access to primary care is limited for the general population. ${ }^{4,21}$ Our population-based sample reduces bias, as it is validated in both urban and rural community settings where the majority of COPD occurs, and incorporates biomass exposure which is a leading risk factor for COPD globally. ${ }^{2}$

Although there has been conflicting data to support application of case-finding instruments in high-income settings, few studies to date have assessed the application of these tools in low- and middle-income settings. ${ }^{22,23}$ Jithoo et al found PEF as a simple and cost effective screening tool for COPD in the BOLD cohort. ${ }^{20}$ COPD diagnosis and treatment is limited by a lack of trained health care providers and spirometry. ${ }^{4,24}$

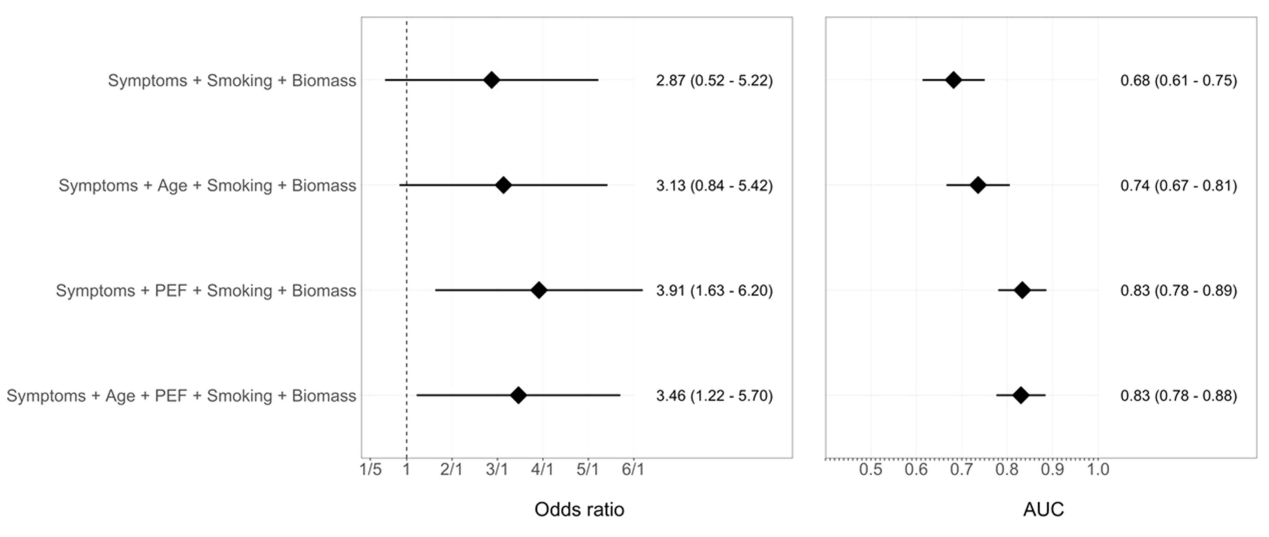

Figure 3 Risk factors for COPD and diagnostic accuracy of demographic and respiratory questionnaires alone, peak expiratory flow (PEF) alone and combined questionnaires with PEF. Odds of COPD (left panel) stratified by age and symptom score, PEF and combined age, symptoms/exposure score and PEF with $95 \%$ Cl. Area under the curve (AUC) for COPD (right panel) stratified by age and symptom score, PEF and combined age, symptoms score and PEF with $95 \% \mathrm{Cl}$. 
Table 3 Operating Characteristics by COLA Score

\begin{tabular}{|l|l|l|l|l|l|}
\hline Score & $\begin{array}{l}\text { Sensitivity } \\
\text { (\%) }\end{array}$ & $\begin{array}{l}\text { Specificity } \\
\text { (\%) }\end{array}$ & $\begin{array}{l}\text { PPV } \\
\text { (\%) }\end{array}$ & $\begin{array}{l}\text { NPV } \\
\text { (\%) }\end{array}$ & PLR \\
\hline $\begin{array}{l}\text { COLA } \\
\geq 2\end{array}$ & 96 & 41 & 7 & 100 & 1.6 \\
\hline $\begin{array}{l}\text { COLA } \\
\geq 3\end{array}$ & 70 & 81 & 15 & 98 & 3.7 \\
\hline $\begin{array}{l}\text { COLA } \\
\geq 4\end{array}$ & 43 & 96 & 32 & 97 & 9.9 \\
\hline $\begin{array}{l}\text { COLA } \\
\geq 5\end{array}$ & 19 & 99 & 50 & 96 & 21.1 \\
\hline
\end{tabular}

Abbreviations: PPV, positive predictive value; NPV, negative predictive value ; PLR, positive likelihood ratio.

In the rural district where the study was conducted, there was no spirometry available for clinical use at time of sampling. As a result, task shifting has been proposed as an effective method for improving diagnosis and treatment of chronic respiratory disease in these settings. ${ }^{25}$ Case-finding instruments provide simple, low-cost and valid tools which can be implemented by local health care professionals and community health workers to identify individuals with likely COPD and refer to specialty clinics for additional testing and management. ${ }^{24}$

This study has several strengths. First, we conducted this study in a population-based, cross-sectional sample. Few patients with respiratory diseases are treated in clinical settings in low- and middle-income countries. Second, we assessed the efficacy of case finding in urban and rural settings among populations with different socioeconomic and exposure histories. Our study also has potential limitations. A major limitation of case-finding instruments, particularly in low- and middle-income settings, pertains to the overlap between COPD and asthma. ${ }^{26}$ Many of the questions identified as having the greatest odds of identifying COPD, are related to asthma. The distinction between asthma and COPD is complex and a pragmatic approach may be the ability to define "fixed airflow obstruction" through case-finding instruments. As such, the diagnostic power of the present instrument is limited (Table 3). Further evaluation and spirometry beyond the COLA would be required for the decision of initiating treatment. Additionally PEF is largely effort dependent. Use of PEF from spirometry data may not be fully extrapolated to clinical situations using standard PEF, particularly in the setting of bronchodilator response. ${ }^{27}$ We needed to exclude 329 participants because of incomplete data, and the overall sample size is limited. Additionally, while daily exposure to cigarette exposure was predictive of COPD in high-income settings, we found exposure assessments did not have as high discrimination for COPD as symptoms and peak expiratory flow. Cigarette exposure in low- and middle-income settings, while increasing, remains low and total pack-years may be difficult to assess in rural settings of Uganda where tobacco exposure in rural areas may reflect smoking of local tobacco crops as opposed to purchased cigarettes. ${ }^{28}$ Assessment of biomass exposure to discriminate COPD was limited due to the ubiquitous use of biomass (wood or charcoal) for cooking in the study area. For the purposes of feasibility of administration, peak expiratory flow measurements were not standardized to height and gender. Lastly, this COPD case-finding instrument will require external independent validation with data pertaining to exacerbation history and COPD disease severity. Further studies across LMIC settings will be required and are in progress to assess the predictive value of these exposure assessments for COPD case finding. ${ }^{24}$

\section{Conclusions}

We developed a seven-item questionnaire, which when combined with age and peak expiratory flow measurements, has high discrimination for COPD in a population-based setting. Whereas respiratory symptom questionnaires were moderately efficacious in predicting airflow obstruction on spirometry, in our study setting, the presence of common respiratory symptoms was highly predictive of COPD. Given the high burden of undiagnosed, symptomatic COPD in LMICs, case-finding instruments may provide a valuable tool in bridging the treatment gap. ${ }^{3}$ Future studies testing the validity of the COLA score across a range of low- and middle-income settings, as well as assessing acceptability and feasibility among lay health workers, are needed to assess effectiveness of case-finding as a public health intervention. ${ }^{29}$

\section{Acknowledgments}

The LiNK study was conducted by Johns Hopkins University in close collaboration with the Makerere Lung Institute and African Community Centre for Social Sustainability (ACCESS) Uganda. The study was supported by the Fogarty International Centre (5R25TW009340) of the US National Institutes of Health, by the COPD Discovery Fund and the Centre for Global Health of Johns Hopkins University. In addition, W.C. is supported under UM1HL134590. T.S. is supported by a Mentored Career Development Award through the National Heart, Lung, and Blood Institute of the NIH 
(K23HL146946). We thank Faith Nassali and Denis Mwanda for making the study possible. We additionally thank the participants of the LiNK cohort study.

\section{Author Contributions}

TS and WC conceptualized the study, obtained funding, and provided overall supervision. TS, BK and WC designed the study in collaboration with RK. TS, ACW, SIP, SH and WC conducted the analyses and interpretation. All authors contributed to data analysis, drafting or revising the article, have agreed on the journal to which the article will be submitted, gave final approval of the version to be published, and agree to be accountable for all aspects of the work.

\section{Disclosure}

Dr Robert A Wise reports personal fees from GSK, Novartis, AstraZeneca, Merck, Anapsysbio, Kikiksa, Kinevant, Galderma, Chimerx, and AbbVie, outside the submitted work. Professor John R Hursts report grants, personal fees, non-financial support from AstraZeneca, Boehringer Ingelheim, Chiesi, and Novartis that make medicines to treat COPD, outside the submitted work. The authors report no other conflicts of interest in this work.

\section{References}

1. Soriano JB, Abajobir AA, Abate $\mathrm{KH}$, et al. Global, regional, and national deaths, prevalence, disability-adjusted life years, and years lived with disability for chronic obstructive pulmonary disease and asthma, 1990-2015: a systematic analysis for the Global Burden of Disease Study 2015. Lancet Respir Med. 2017;5(9):691-706. doi:10.1016/S2213-2600(17)30293-X

2. Siddharthan T, Grigsby MR, Goodman D, et al. Association between household air pollution exposure and chronic obstructive pulmonary disease outcomes in 13 low-and middle-income country settings. Am J Respir Crit Care Med. 2018;197(5):611-620. doi:10.1164/rccm.2017091861OC

3. Lamprecht B, Soriano JB, Studnicka M, et al. Determinants of underdiagnosis of COPD in national and international surveys. Chest. 2015;148(4):971-985. doi:10.1378/chest.14-2535

4. Robertson NM, Nagourney EM, Pollard SL, et al. Urban-rural disparities in chronic obstructive pulmonary disease management and access in Uganda. Chronic Obstr Pulm Dis. 2019;6(1):17. doi:10.15326/jcopdf.6.1.2018.0143

5. Martinez FJ, Mannino D, Leidy NK, et al. A new approach for identifying patients with undiagnosed chronic obstructive pulmonary disease. Am J Respir Crit Care Med. 2017;195(6):748-756. doi:10.1164/rccm.201603-0622OC

6. Martinez FJ, Raczek AE, Seifer FD, et al. Development and initial validation of a self-scored COPD population screener questionnaire (COPD-PS). COPD: J Chron Obstruct Pulmon Dis. 2008;5 (2):85-95. doi:10.1080/15412550801940721

7. Yawn BP, Mapel DW, Mannino DM, et al. Development of the lung function questionnaire (LFQ) to identify airflow obstruction. Intl J COPD. 2010;5:1-10.

8. Siddharthan T, Grigsby M, Morgan B, et al. Prevalence of chronic respiratory disease in urban and rural Uganda. Bull World Health Organ. 2019;97(5):318-327. doi:10.2471/BLT.18.216523
9. National population and housing census 2014. Main report. Kampala: Uganda Bureau of Statistics; 2014. Available from: https://www. ubos.org/onlinefiles/uploads/ubos/NPHC/NPHC\%202014\%20FINAL \%20RESULTS\%20 REPORT.pdf. Accessed December 13, 2017.

10. Statistical Abstract. Kampala: Uganda Bureau of Statistics; 2001.

11. Hankinson JL, Kawut SM, Shahar E, et al. Performance of American thoracic society-recommended spirometry reference values in a multiethnic sample of adults: the multi-ethnic study of atherosclerosis (MESA) lung study. Chest J. 2010;137(1):138-145. doi:10.1378/ chest.09-0919

12. Hankinson JL, Odencrantz JR, Fedan KB. Spirometric reference values from a sample of the general US population. Am J Respir Crit Care Med. 1999;159(1):179-187. doi:10.1164/ajrccm.159.1.9712108

13. Miller MR, Hankinson J, Brusasco V, et al. Standardisation of spirometry. Eur Respir J. 2005;26(2):319-338. doi:10.1183/ 09031936.05.00034805

14. Breiman L. Random forests. Mach Learn. 2001;45(1):5-32. doi:10.1023/A:1010933404324

15. Firth D. Bias reduction of maximum likelihood estimates. Biometrika. 1993;80(1):27-38. doi:10.1093/biomet/80.1.27

16. Hosmer DW, Lemeshow S. Goodness of fit tests for the multiple logistic regression model. Commun Stat. 1980;9:1043-1069. doi:10.1080/03610928008827941

17. Rivera R, Cosio M, Ghezzo H, et al. Comparison of lung morphology in COPD secondary to cigarette and biomass smoke. Int $J$ Tuberc Lung Dis. 2008;12(8):972-977.

18. Siddharthan T, Gupte A, Barnes PJ. COPD endotypes in low-and middle-income country settings: precision medicine for all. $\mathrm{Am}$ $J$ Respir Crit Care Med. 2020;202(2):171-172. doi:10.1164/ rccm.202001-0165ED

19. Hanania NA, Mannino DM, Yawn BP, et al. Predicting risk of airflow obstruction in primary care: validation of the lung function questionnaire (LFQ). Respir Med. 2010;104(8):1160-1170. doi:10.1016/j. rmed.2010.02.009

20. Jithoo A, Enright PL, Burney P, et al. Case-finding options for COPD: results from the burden of obstructive lung disease study. Eur Respir J. 2013;41(3):548-555. doi:10.1183/09031936.00132 011

21. Mills A. Health care systems in low-and middle-income countries. $N$ Engl J Med. 2014;370(6):552-557. doi:10.1056/NEJMra1110 897

22. Guirguis-Blake JM, Senger CA, Webber EM, et al. Screening for chronic obstructive pulmonary disease: evidence report and systematic review for the US preventive services task force. JAMA. 2016;315 (13):1378-1393. doi:10.1001/jama.2016.2654

23. Soriano JB, Zielinski J, Price D. Screening for and early detection of chronic obstructive pulmonary disease. Lancet. 2009;374 (9691):721-732. doi:10.1016/S0140-6736(09)61290-3

24. Siddharthan T, Pollard SL, Quaderi SA, et al. Effectivenessimplementation of COPD case finding and self-management action plans in low-and middle-income countries: global excellence in COPD outcomes (GECo) study protocol. Trials. 2018;19(1):571. doi:10.1186/s13063-018-2909-8

25. Siddharthan T, Ramaiya K, Yonga G, et al. Noncommunicable diseases in East Africa: assessing the gaps in care and identifying opportunities for improvement. Health Aff. 2015;34(9):1506-1513. doi: $10.1377 /$ hlthaff.2015.0382

26. Morgan BW, Grigsby MR, Siddharthan T, et al. Epidemiology and risk factors of asthma-chronic obstructive pulmonary disease overlap in low-and middle-income countries. $J$ Allergy Clin Immunol. 2019;143(4):1598-1606. doi:10.1016/j.jaci.2018.06.052

27. Thiadens H, De Bock G, Van Houwelingen J, et al. Can peak expiratory flow measurements reliably identify the presence of airway obstruction and bronchodilator response as assessed by FEV1 in primary care patients presenting with a persistent cough? Thorax. 1999;54(12):1055. doi:10.1136/thx.54.12.1055 
28. van Gemert F, Kirenga B, Chavannes N, et al. Prevalence of chronic obstructive pulmonary disease and associated risk factors in Uganda (FRESH AIR Uganda): a prospective cross-sectional observational study. Lancet Global Health. 2015;3(1):e44-e51. doi:10.1016/S2214109X(14)70337-7
29. van Boven JF. Costs of case-finding uncovered: time to revisit COPD's value pyramid? BMJ Publishing Group Ltd; 2019.

\section{Publish your work in this journal}

The International Journal of COPD is an international, peer-reviewed journal of therapeutics and pharmacology focusing on concise rapid reporting of clinical studies and reviews in COPD. Special focus is given to the pathophysiological processes underlying the disease, intervention programs, patient focused education, and self management protocols. This journal is indexed on PubMed Central, MedLine and CAS. The manuscript management system is completely online and includes a very quick and fair peer-review system, which is all easy to use. Visit http://www.dovepress.com/testimonials.php to read real quotes from published authors.

Submit your manuscript here: https://www.dovepress.com/international-journal-of-chronic-obstructive-pulmonary-disease-journal 